https://www.journal-imab-bg.org

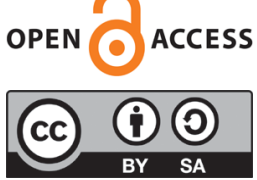

Original article

\title{
ASSESSMENT OF KNEE JOINT POSITION SENSE IN PATIENTS WITH MULTIPLE SCLERO- SIS
}

\author{
Miglena Tsvetkova-Gaberska ${ }^{1}$, Nevena Pencheva ${ }^{2}$ \\ 1) Department of Kinesitherapy, Faculty of Public Health, Health Care and \\ Sports, SWU “Neofit Rilski”, Blagoevgrad, Bulgaria \\ 2) Department of Anatomy and Physiology, Faculty of Public Health, Health \\ Care and Sport, University Center for Functional Research in Sports and Ki- \\ nesitherapy, South-West University "Neofit Rilski", Blagoevgrad, Bulgaria.
}

\section{ABSTRACT:}

Purpose: Multiple sclerosis (MS) disrupts joint position sense (JPS). The aims were: - to assess the JPS of knee extensors, using three target angles in MS patients and in healthy; - to estimate the changes in reproducing the joint position by using absolute (AE), constant (CE) and variable error (VE) and the effect of sex on knee JPS acuity.

Subjects and Methods: Twenty MS patients and twenty healthy were tested, by knee joint angle reproduction with three targets $\left(20^{\circ}, 60^{\circ}, 75^{\circ}\right)$, by passive and active mode, without visual and verbal feedback. JPS measurement was done using isokinetic dynamometry. Deviations from the targets were calculated as AE, VE and CE, which correspond to accuracy, precision and direction of the bias, respectively.

Results: Increased values of $\mathrm{AE}$ and sex differences in JPS accuracy in MS individuals, as compared with healthy, were: - at targets $20^{\circ}$ and $60^{\circ}$ in females in passive mode in both legs; - at $60^{\circ}$ in the involved leg in males (more pronounced as compared with females); and - at $75^{\circ}$ in the less involved leg, in passive reproduction in women and active in men. The significantly increased negative CE indicates a more pronounced deficit in males with MS at $60^{\circ}$ in the involved leg and at $75^{\circ}$ in less involved leg. Increased VE in passive repositioning was also proved $(\mathrm{p}<0.05)$ in females with MS at $20^{\circ}$ and $60^{\circ}$.

Conclusion: These results suggest impaired proprioceptive performance and sex differences in MS individuals. The findings provide information to recommend appropriate strategies for the evaluation and treatment of proprioceptive deficits.

Keywords: proprioception, joint position sense, isokinetic dynamometry, knee joint, multiple sclerosis.

\section{INTRODUCTION}

Multiple sclerosis (MS) is a degenerative disease, which disrupts motor control of the skeletal muscles, but most people, especially with relapsing-remitting type, live with MS for decades after their diagnosis. Sensory problems, disturbances in feeling, and proprioceptive deficits are often one of the earliest symptoms of MS, which are followed by falls and gait impairments in their daily life [1].

As a substantial part of the proprioception, joint position sense (JPS) represents the ability of the subject to actively or passively reproduce a previously perceived joint angle, i.e. limb position, in the absence of visual feedback. The description of this type of proprioceptive senså, in fact, presents the method for its evaluation, called angle reproduction or repositioning test. Although this method has been adopted preferentially for use in studies [2, 3, 4, 5], there is insufficient data in the pertinent literature, presenting results on patients with MS, by means of angle reproduction test, which cover more detailed aspects, such as differences between passive or active repositioning techniques, comparisons with less involved limb, sex differences, etc., although some of these approaches are difficult to implement as a clinical trial.

Comparing results obtained from healthy persons, Laufer et al. [2] suggest that the differences between proprioceptive performance following passive and active reproduction test relate to a contribution of central control, i.e. the role of the feedback, provided by receptors sensitive to joint position, and of the feed forward mechanism as the second way of participation of the proprioceptive signaling. So, this experimental design, applied in subjects with MS, would be quite informative for the evaluation and treatment of proprioceptive deficits.

Knee joint proprioception seems to be of particular importance in patients with MS, as it provides the most stable postural position. Knee extensors are vital muscles in keeping the knee joint stable in the upright position and in various forms of locomotion [3].

Sex differences in the knee joint position sense acuity are the object of negligence in patients with MS and contradictory results, and in many studies, groups are mixed $[3,4]$. There are data on such differences in healthy individuals found in the study of Vafadar et al.[5], for shoulder joint position sense and in our previous investigation for knee force sense [6].

The commonly used joint position reproduction test 
protocol is a form of psychophysical experiment. The standardized assessment, using isokinetic dynamometry, is a referent, widely applied methodology [7]. According to the so-called method of adjustment [8], a measure of sensitivity is the difference between the adjustable stimulus and the reference one, which is recorded and calculated as a participant's error. Using of the descriptive statistics allow obtaining of three type of errors - absolute error (AE), constant error (CE) and variable error (VE). They are dependent variables and assess different features of JPS.

Based on the arguments presented, the aims of these study were: - after preliminary anthropometric measurements, to assess the JPS of knee extensors, by means of angle reproduction test, using three target angles in active and passive repositioning, by isokinetic dynamometer in MS patients - males and females, and to compare them with healthy individuals; - to estimate the changes in accuracy, the direction of the bias and precision in reproducing the joint position, by using AE, CE and VE; and - to investigate, the effect of sex on knee JPS acuity.

\section{MATERIALS AND METHODS:}

\section{Participants:}

The research was conducted at the University Center for Functional Research in Sport and Kinesitherapy. A total of 40 subjects were enrolled: 20 patients with MS and 20 healthy controls (HC), subdivided into male and female sex subgroups. The healthy persons were required to be free of organic diseases, to have normal vision and normal joint range of motion of the knee joint on the dominant side. The inclusion criteria for the patients were as follows: men and women with relapsing-remitting MS, aged 30-50 years and values for Expanded Disability Status Scale (EDSS) d"3, indicating that the disablement was not severe enough to impair their full daily and work activities. Subject recruitment and the experimental procedures were approved by the Ethics Committee of South-West University of Blagoevgrad (No 1901-2, 2019). All participants were informed verbally for the purpose of the study, the procedures and protocols to be implemented, and signed a written informed consent.

\section{Measurement procedures:}

The basic anthropometric parameters of all participants were measured by a body composition analyzer machine (Ioi 353). The measurement was conducted in the morning on an empty stomach and bladder, under the prescribed conditions, related with the food and liquid intake and exercise.

Before we collect the data for the joint angle reset test, all participants performed a general warm-up, consisting of a 5 min cycling with a stationary bike with a load of about $120 \mathrm{~W}$ at $60 \mathrm{rpm}$. It was required to optimize the inherent viscoelastic properties of the muscles, tendons and joints of the lower limbs and to prepare the body for the upcoming measurement. Further, the subjects were tested with the joint angle reset method with the Biodex Isokinetic Dynamometer (Biodex, 4S Pro), which was used for measuring the knee proprioception (joint position sense) error of the knee extensor muscles. In healthy persons, the knee joint was tested only at the dominant leg. Since the leg dominance in MS patients is disturbed, their examination was bilaterally (involved and less involved leg). The dynamometer was positioned at $90^{\circ}$ and tilted at $0^{\circ}$. The seat orientation was fixed at $90^{\circ}$, while the seatback tilted at $70^{\circ}-85^{\circ}$. Each subject was asked to sit on the chair, following the instructions to take a comfortable position in order to achieve the best possible test. So, the additional adjustments of the seat area length, back angle and seat height optimized each individual's position. Then the subject was secured with snug belts across the shoulder, chest and hip of the tested leg. The rotational axis of the knee joint was aligned with the dynamometer rotational axis. The cuff of the dynamometer's lever arm, called knee attachment, is wrapped tightly around the lower leg above the level of the lateral ankle malleoli.

The joint angle reproduction method was applied by active reproduction mode with voluntary movements and passive reproduction mode - by means of a motor drive. The subjects were required to experience and then reproduce joint positions in both cases, from start position $90^{\circ}$. Several target angles, very mild flexion $\left(20^{\circ}\right)$, intermediate to strong flexion $\left(60^{\circ}\right)$ and very strong flexion $\left(75^{\circ}\right)$, were used. These angles are commonly achieved during the phases of lifting and placing the legs when walking and other daily activities. It was conducted preliminary training to generate subjective positional sensation at a given level of knee extension, as well as the ability to visually follow up the graphical interface of the monitor and familiarize himself/herself with the so-called hold button on the dynamometer. Each subject was trained with and without visual and verbal feedback before the real measurement. The measurement had the following algorithm: (1) the dynamometer passively moved the joint with an angular velocity at approximately $0.5 \% \mathrm{sec}$ to the test position three times with and three times without visual and verbal feedback; (2) the subject attempted to identify (sense) the test position whilst holding it actively (isometrically) for approximately ten seconds (a time period sufficient to allow the subject to remember the target position); (3) the dynamometer passively returned the joint to the starting position; then (4) the subject attempted to actively reproduce the previous position and to press the hold button.

First, the participants made actively three measurements at $20^{\circ}$. After that, while they still remembered the target, they made a passive protocol at the same angle. The protocol was the same for $60^{\circ}$ and $75^{\circ}$. The passing from active to passive was $2 \mathrm{~min}$. In the protocol for passive angle reproduction, the dynamometer moved the limb passively, and the participant had to press the hold button when reaching the target angle in the knee joint. During the test, subjects were asked to relax as much as possible the muscles on the tested limb. The rest between the angular positions was 5 min when the subject is releasing from the belts and is able to move the examined limb freely.

\section{Calculation of the proprioceptive acuity:}

The value of individual error in each participant's attempt was determined through the difference between the position reproduced and the position experienced. We es- 
timated these differences using indicators of descriptive statistics, which appears dependent variables. Thus, we determined the proprioceptive acuity by means of the values of the $\mathrm{AE}, \mathrm{VE}$ and $\mathrm{CE}$, because each type characterizes the separate feature of the performance. The calculation of each variable is described in our previous study in detail [6]. Briefly, the absolute deviations from a target are reported as AE. They are obtained through the arithmetic mean of the individual errors in the modulus (because it neglects the direction of the error) and determine the individual's accuracy in reproducing the joint position. The $\mathrm{CE}$ is a measure of the direction of the mismatch or bias from the target joint position and could be with a positive or negative value. As an arithmetic means of the individual errors with their signs, CE determines the tendency for the position to be reproduced above (overestimated) or below (underestimated) the target. As a descriptive statistic parameter, the VE is the standard deviation from the target angle of the individual errors and could be a criterion for precision and consistency of the reproductions made. So, VE provides information for the within-subject variability. Although the VE serves as an indicator of variability of the performance, it provides no information of the performance relative to the target.

\section{Statistical analysis:}

Data were analyzed by using Graph Pad Prism (Ver.
3.0). All anthropometric indices, indexes and errors are presented as means with standard deviations (SD) and coefficient of variation, obtained by descriptive statistics. To measure the differences between mean values, the nonparametric test of Mann-Whitney, the Kruskal-Wallis ANOVA test and Dunn's post hoc test were applied $[6,9]$. The statistical significance level established was $\mathrm{p} \leq 0.05$.

\section{RESULTS:}

According to the anthropometric characteristics of the subjects from both groups, presented in Table 1, it was found that: (a) there were no significant differences between the averages for age groups of men and women in healthy subjects and in patients; therefore, comparative analyzes between the groups are relevant and not influenced by age differences; (b) in all other anthropometric variables, average values for women were significantly lower $(\mathrm{p}<0.05)$ than those of men, which is well known, except for the percentage of the mass of body fat of women with MS, which was significantly higher than that of men, while in healthy women it was insignificantly lower than that in healthy men; (c) values of body mass index (BMI) in women with MS were the lowest among all responders (healthy and patients), but their percentage of the mass of body fat was highest, and the increase in relative percent in men with MS, was statistically significant $(\mathrm{p}<0.05)$.

Table 1. Anthropometric characteristics (mean \pm SD) of healthy controls and patients with multiple sclerosis (MS).

\begin{tabular}{|l|cc|cc|}
\hline \multirow{2}{*}{ VARIABLE } & \multicolumn{2}{|c|}{ HAELTHY CONTROLS } & \multicolumn{2}{c|}{ PATIENTS WITH MS } \\
\cline { 2 - 5 } & $\begin{array}{c}\text { MALES } \\
(\mathbf{n}=\mathbf{1 0})\end{array}$ & $\begin{array}{c}\text { FEMALES } \\
(\mathbf{n}=\mathbf{1 0})\end{array}$ & $\begin{array}{c}\text { MALES } \\
(\mathbf{n}=7)\end{array}$ & $\begin{array}{c}\text { FEMALES } \\
(\mathbf{n}=\mathbf{1 3})\end{array}$ \\
\hline Age (years) & $42.1 \pm 8.0$ & $43.3 \pm 7.4$ & $44.2 \pm 6.9$ & $42.1 \pm 8.1$ \\
Height $(\mathrm{cm})$ & $173.0 \pm 5.8$ & $166.2 \pm 4.8^{*}$ & $175.7 \pm 8.7$ & $163.8 \pm 6.7 *$ \\
Body mass $(\mathrm{kg})$ & $84.6 \pm 12.2$ & $64.3 \pm 10.2^{*}$ & $85.2 \pm 16.3$ & $61.1 \pm 19.6 *$ \\
Body mass index $\left(\mathrm{kg} / \mathrm{m}^{2}\right)$ & $28.2 \pm 3.3$ & $23.3 \pm 3.6^{*}$ & $27.5 \pm 4.2$ & $22.6 \pm 6.1 *$ \\
Lean body mass $(\mathrm{kg})$ & $61.4 \pm 6.0$ & $46.5 \pm 3.8^{*}$ & $62.5 \pm 8.1$ & $44.5 \pm 7.1 *$ \\
Mass of body fat $(\%)$ & $27.3 \pm 4.4$ & $26.5 \pm 7.4$ & $25.9 \pm 7.6$ & $28.2 \pm 8.1 *$ \\
\hline
\end{tabular}

Designation: *Statistically significant difference $(\mathrm{p}<0.05)$ as compared to males (Mann Whitney test).

Concerning the comparison between both active and passive repositioning, we found in healthy responders, in all types of errors, that the judgements in active mode for the knee joint position were better than passive judgements in target angle $20^{\circ}$ and significantly worse than passive judgements in target angles $60^{\circ}$ and $75^{\circ}$. This tendency was statistically significant for $\mathrm{AE}$ and $\mathrm{CE}$ in targets $20^{\circ}$ and $60^{\circ}$ and for $\mathrm{VE}$ in targets $60^{\circ}$ and $75^{\circ}$. The same pattern also occurred in patients with MS with the exception of men in target angle $20^{\circ}$ for AE and CE, where the accuracy in passive mode was better, as compared with active.

The analysis of the results obtained for the mean values of $\mathrm{AE}$ and for the significance of the differences between MS patients and healthy, in both modes of application (active and passive) of the joint angle reproduction test (Kruskal-Wallis ANOVA test, Dunn's test), showed the following: (a) At a target angle of $20^{\circ}$, AE values, both in the involved and less involved leg in women with MS, increased significantly in the passive mode, as compared with $\mathrm{HC}$ (Fig. 1, Aa); no significant differences in AE were observed at target angle $20^{\circ}$ in men with MS compared to healthy individuals in the two reproduction modes (Fig. 1, $\mathrm{Ba})$;

(b) The accuracy of the reproduction of target angle $60^{\circ}$ in women with MS was also reduced because the values of AE significantly increase compared to healthy women, and this refers to the passive mode and affects both legs (Fig. $1 \mathrm{Ab}$ ); a significant elevation in the values of $\mathrm{AE}$ at target $60^{\circ}$ was also observed in men with MS, but it concerns the active mode and only the knee joint of the more damaged (involved) leg (Fig. 1, Bb); the comparison between men and women with MS in the same target angle at the involved leg showed significantly higher AE values 
in men (Fig. 1, Ba/Bb); and

(c) It was found reduced accuracy in reproducing a target angle of $75^{\circ}$ (strong flexion) in the less involved leg of patients with MS, which was manifested by significantly increased $\mathrm{AE}$ values in passive reproduction in women (Fig. $1, \mathrm{Ac})$ and active in men (Fig. 1, Bc).

Fig. 1. Knee joint position sense presented as an absolute error at three target knee angles $-20^{\circ}, 60^{\circ}$ and $75^{\circ}$ in active and passive reproduction mode in females - $\mathbf{A}$ and in males $-\mathbf{B}$.

A
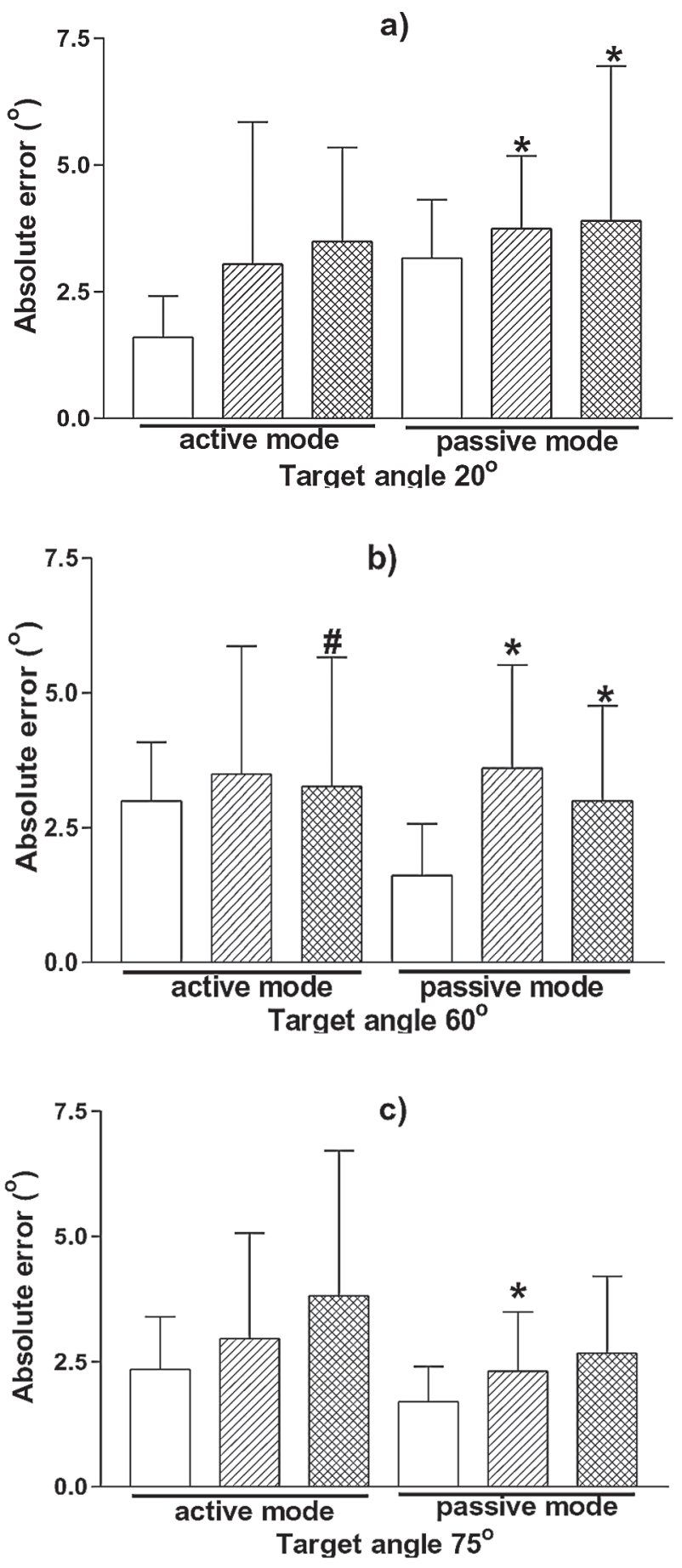

B

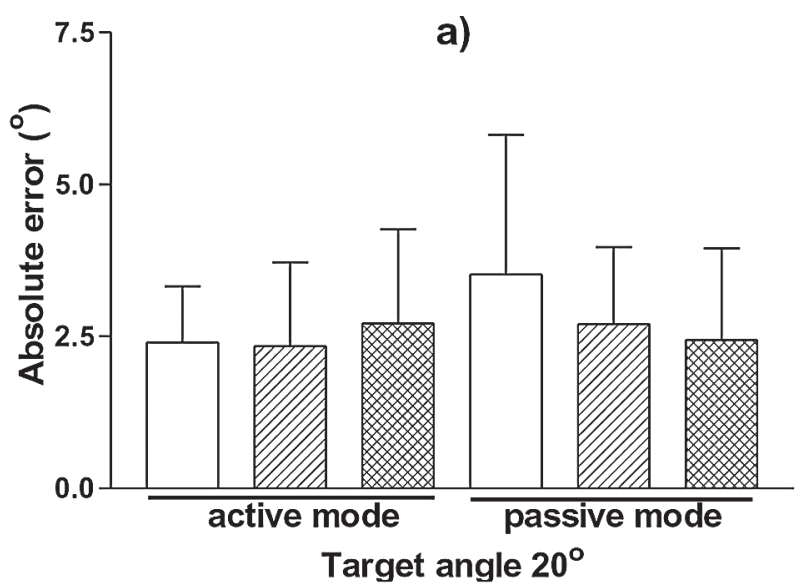

b)

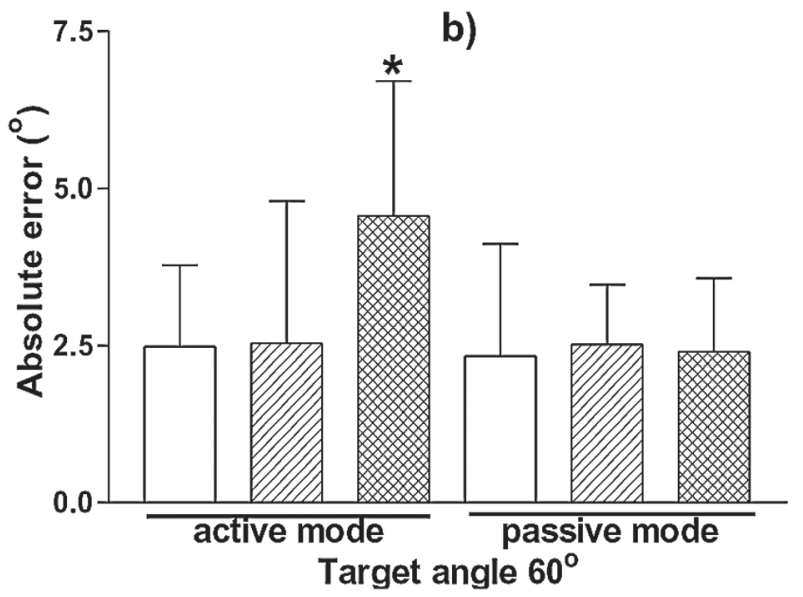

c)

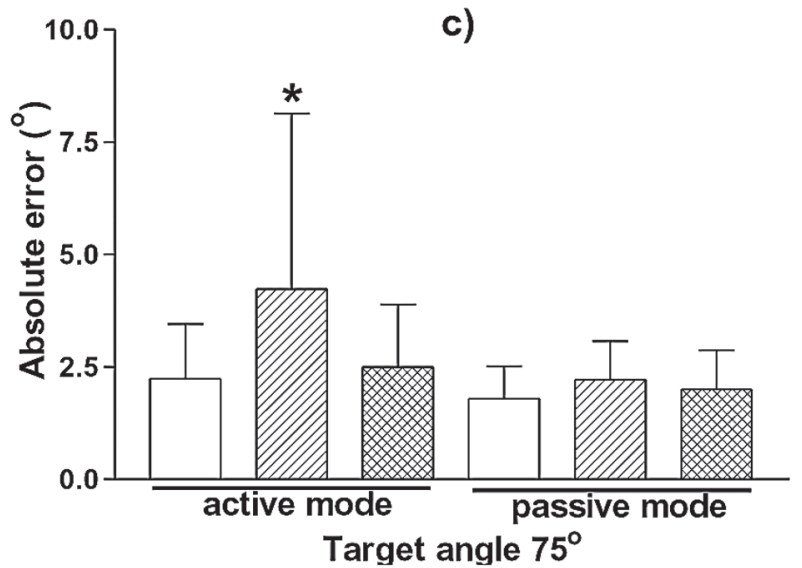

Designations:

Dominant Leg - Controls Less Involved Leg -MS Involved Leg-MS

* Statistically significant difference as compared to healthy subjects from the same testing mode $(p<0.05)$,

\# Statistically significant difference as compared to men from the same testing mode (Kruskal-Wallis ANOVA test, Dunn's test, selected pairs, $p<0.05$ ) 
Attributes of the CE are the direction and the magnitude of the deviations from the target angles. It was established that in healthy men and women, the direction of deviation depends on the degree of flexion, which is determined by the value of the target angle (data not shown). It was found that in mild flexion $\left(20^{\circ}\right)$, the target angle in active mode is reproduced exactly because $\mathrm{CE} \approx$ 0 (regardless of direction), while the target angles in moderate and strong flexion $\left(60^{\circ}\right.$ and $\left.75^{\circ}\right)$ were underestimated. The described dependence in $\mathrm{HC}$ also occurred in the active reproduction mode, but the range of deviations started from overestimation, and at target, $75^{\circ}$ was underestimated. In patients with MS, the relationship between the direction of deviations and the degree of flexion is preserved, but in men only, in active mode, significantly higher values of CE were observed, as compared with healthy subjects, when reproducing the target $60^{\circ}$ in the involved leg $\left(\mathrm{CE}_{\mathrm{HC}}=-1.2 \pm 1.9 ; \mathrm{CE}_{\mathrm{MS}}=-4.3 \pm 2.1\right)$ and target $75^{\circ}$ for less involved leg $\left(\mathrm{CE}_{\mathrm{HC}}=-1.2 \pm 1.3 ; \mathrm{CE}_{\mathrm{MS}}=-\right.$ $2.5 \pm 1.4)$. These results are evidence for the more pronounced proprioceptive deficit in males with MS, as far as JPS is concerned.

The analysis of the data for the VE, which defines the precision of reproduction of the three target angles, proved a statistically significant increase $(p<0.05)$ in the values, which applies only to the group of women with MS in passive mode, compared to healthy controls, for a target of $20^{\circ}\left(\mathrm{VEHC}_{\mathrm{HC}}=2.3 \pm 1.5 ; \mathrm{VE}_{\mathrm{MS}}=3.7 \pm 2.1\right)$ and a target of $60^{\circ}\left(\mathrm{VE}_{\mathrm{HC}}=1.2 \pm 0.8 ; \mathrm{VE}_{\mathrm{MS}}=2.5 \pm 1,3\right)$ in the less involved leg.

\section{DISCUSSION:}

Deficiencies in proprioception are known to cause disturbances in mobility, gait and balance, falls, injuries, and this reduces the quality of life. The present study aims to assess quantitative parameters of proprioceptive perception of the position of the knee joint extensors in patients with MS and to compare them with healthy people since evaluating proprioception is relevant to rehabilitation because of its significance in motor control.

JPS refers to the ability to reproduce a given joint angle by active or passive mode, and this design allows to assess of three types of errors of the deviations measured, which describe different features of this proprioception sense. The sex division of the responders allowed us to identify significant sex differences in the reproduction of three target angles in the knee joint. The obtained results show significant differences between patients with MS and healthy individuals, which are more pronounced in men with MS in some aspects.

In anthropometric measurements, no differences were found between patients and healthy individuals, similar to Wens et al. [10], with the exception of significantly higher values of the mass of body fat percentage in women with MS compared to men, which is not observed in healthy people. According to Dionyssiotis [11], the values of BMI in MS patients often underestimate body fat and may mask adiposity. This is in accordance with our data for low values of BMI but a very high percentage of the mass of body fat in women with MS. However, this underestimation of body fat through BMI in people with neurologic disabilities could probably be due to degenerative loss of muscle mass rather than obesity. This tendency is less pronounced in men with MS, which could be related to the androgens, for which there is evidence in the literature [12].

The comparison between the active and passive mode of reproduction in all responders and for the three types of errors in the present study favors the accuracy of the performance in active mode at $20^{\circ}$ and in passive at $60^{\circ}$ and $75^{\circ}$. There are contradictory data in the literature on the importance of various factors on the performance, following active versus passive repositioning in the application of the joint position reproduction test. The results can be interpreted in at least two ways: - one is related to muscle length at the end of the movement at different target angles, which is evidenced in studies of the correlation between JPS and sense of muscle force, which are signaled by muscle spindles $[11,13,14]$; - the other way concerns the role of the length of the distance traveled by the limb under test at different target angles [2]. It is known that proprioceptive information about body and limb position participates before initiation of the movement (as a basis for programming of the motor commands), so during movement (modulating of the motor commands) and finally towards movement completion, by proprioceptive feedback for final corrections [2]. It can be suggested that the better proprioceptive performance in active mode in target $20^{\circ}$ is due to the longer period of modulation of motor commands during proprioceptive input on limb progression in volitional movement. Obviously, additional targeted experiments in healthy individuals are needed to clarify these findings.

The results obtained on the average errors in all three species represented a complex approach to the assessment of changes in sensitivity of JPS in MS patients compared to healthy subjects.

The absolute error presents in the most comprehensive version of the results of the study of knee joint position sense. It determines the accuracy of the task and shows a statistically significant increase in the values in MS patients at all target positions. At the $20^{\circ}$ joint position in the passive reproduction mode, a decrease in accuracy was observed for both less involved and involved leg only in the group of women with MS compared to HC. The same tendency for a statistical increase of AE or lower accuracy of JPS execution is also present at positions $60^{\circ}$ and $75^{\circ}$ again in passive mode for both legs. In men, higher values of AE compared to healthy individuals are at $60^{\circ}$ and $75^{\circ}$, but in the mode of active reproduction. This is most likely due to the level of disability in both groups and more pronounced deficits in men in active mode. On the other hand, accuracy in men is reduced as a result of impaired mechanoreceptors irritation during muscle fibre elongation. In women, muscle elongation in active mode signals correctly, so they do not show significant deviations from the data of healthy individuals. According to Neamtua et al. [15], active protocols should 
lead to better results because, during the testing period, more mechanoreceptors were stimulated than passive protocols for JPS measurement. In addition, Stillman [4] provides evidence that protocols with active reproduction in JPS lead to lower AE values, most likely due to increased muscle activation of mechanoreceptors as a result of increased muscle contraction in the knee joint. In a study by Guney et al. [16], while the eccentric force correlates with the two target angles of JPS $\left(20^{\circ}\right.$ and $\left.60^{\circ}\right)$, the concentric force correlates only with $20^{\circ}$ in patients with patellofemoral syndrome. This shows that the role of muscle antagonists in the joint is extremely important for the accuracy of JPS performance. At positions $60^{\circ}$ and $75^{\circ}$, there are differences only in passive mode for the less injured and injured leg in women. In male patients, the largest deviation from $\mathrm{AE}$ values is $60^{\circ}$ active for the injured leg, and this is most likely related to the measured isometric force deficit in $60^{\circ}$ and the more manifested gait disorders [6]. In healthy individuals, this angular position of the lower limb contributes to the relative shortening of the swinging leg so that it passes through the supporting leg without the foot stepping on the floor. Moghadasi et al. [17] examined knee JPS at $30^{\circ}$ and $60^{\circ}$ after 8 weeks of resistive TRX training in patients with relapsing-remitting MS. The authors demonstrated a statistical reduction in $\mathrm{AE}$ at only $60^{\circ}$ in the non-dominant leg compared to the control group. According to them, this angular position is associated with the conduction and transmission of proprioceptive information at the level of the spine, which increases postural fluctuations, slows down the reactions of the support and manifests diseasespecific imbalances. This somatosensory dysfunction leads to deficits in postural control and gait and significantly increases the risk of falls $[1,4,18]$. On the other hand, it could have an impact in the form of MS. Kalincik et al. [19] reported a higher incidence of relapses observed in women than in men with relapsing-remitting MS throughout the disease and in all age groups. In summary, the team proved that women are more susceptible to MS and have a more recurrent course of the disease, but men have a faster progression of the disease and subsequent disability. Recent studies have considered the use of proprioceptive and resistive exercises as a potential treatment and retardation of the progression of relapsing-remitting MS [10].

The constant error shows how the direction of deviations from the target, whether below or above the target angle. A statistically significant increase in error was observed only in men in the angular position $60^{\circ}$ and $75^{\circ}$ active reproduction. There are a tendency for both healthy and patients to have negative error values, and this is most characteristic of the active mode of reproduction, i.e. men with MS are most inaccurate with respect to CE in angular positions that do not cause severe irritation and activity of the musculoskeletal mechanoreceptors (Golge tendon organ and muscle spindles) [5]. This is also typical for the active compared to the passive mode. Neguesi et al. [20] studied healthy women, dividing them by age 20-32 and 55-87 and found that older females have (-) CE values, while young (+), as the conclusions are associated with degenerative changes. In our studied groups, the damage is most likely greater in men than in women, which is confirmed by the AE data. On the other hand, the authors compare the occurrence of deficits typical of the elderly over 60 years and people with MS based on degenerative changes as a result of immobility and reduced physical activity [10].

The variable error represents how the reproduction of the target varies. The results show that in men with MS in both passive and active mode, the deviations are smaller, while in women with MS, the values are higher than those of healthy individuals, and their accuracy is impaired. Variability is a general characteristic of human movement that can play a role in the central organization of volitional movement [18, 19]. This is also the trend for healthy subjects. The larger deviations in women with MS and the lack of such in men are most likely due to a number of mechanisms described in the literature. Females have different biomechanics, gait, structural and morphological properties of tendons and joints compared to men. The anterior cruciate ligament in women is smaller than that in men. Women also have less cartilage volume than men, where the percentage difference varies from $19.9 \%$ in the patella to $46.6 \%$ in the medial part of the tibia. Therefore, the number of receptors in the knee joint is probably lower in women than in men [10]. Based on other studies in the literature and analysis of the results, it can be assumed that both healthy and MS females have larger deviations in VE. This makes them more susceptible to intra-articular trauma and injury than men, and despite the disease, some sex differences found by other authors are confirmed [19]. On the other hand, VE in healthy women shows higher values with age and the degenerative changes as a result of the disease and a prerequisite for musculoskeletal injuries [17].

Previous studies have shown that imbalances in people with MS are primarily the result of deficiencies in proprioception. Because MS affects the ability of nerves to conduct information, problems with the transmission of proprioceptive information from the joints of the lower extremities to the brain (and vice versa) may play a key role in impaired balance control [1, 18]. The results of a relatively new study of position perception between patients with MS and a control group using goniometry showed that there were no significant differences in the perception of knee position in both study groups. They conclude that JPS is not a consequence of MS disease, and many factors can influence errors in reproducing the target angle in the knee joint $[3,18]$. Contrary to their study, our results showed significant deficits in target angle reproduction in patients with relapsing-remitting MS and sex differences in knee JPS.

It could be suggested that the isokinetic dynamometry allows test standardization, velocity control, passive and active repositioning modes and etc. This allows a safe, accurate and reliable assessment of both aspects of JPS and to identify the various deficits. 


\section{CONCLUSION:}

In conclusion, the findings of the present study established: (a) a reliable trend for high percentage of body fat mass, but against the background of low values of BMI, proved in females with MS; (b) better accuracy of the proprioceptive performance in active mode of repositioning in the application of joint position reproduction test for knee extensors, at target $20^{\circ}$, while at target $60^{\circ}$ and $75^{\circ}$ in passive mode, which concerns all responders - healthy and MS patients; further study is necessary to determine to what extent this finding is manifested in healthy; (c) impairments in knee joint position sense accuracy in MS individuals as compared with healthy, which reveal sex differences such as reduced accuracy at target $20^{\circ}$ and $60^{\circ}$ in females with MS in passive mode in both legs, at $60 \mathrm{o}$ in involved leg in males (more pronounced as compared with females) and at $75^{\circ}$ in less involved leg, in passive reproduction in women and active in men; and (d) significantly increased negative deviations from the target joint angles (as CE), which indicates more pronounced proprioceptive deficit in males with MS at target $60^{\circ}$ in involved leg and at target of $75^{\circ}$ in less involved leg; additionally decreased precision in passive repositioning was also proved in females with MS for target $20^{\circ}$ and $60^{\circ}$.

These results, obtained by passive and active reproduction mode and calculations of three types of average errors, will provide information necessary to recommend appropriate strategies for the evaluation and treatment of proprioceptive deficits in individuals with MS.

\section{Acknowledgements:}

The study was funded by the National Science Program "Information and Communication Technologies for Unified Digital Market in Science, Education and Security" (2018-2022), supported by the Ministry of Education and Science.

\section{REFERENCES:}

1. Brandstadter R, Ayeni O, Krieger SC, Harel NY, Escalon MX, Katz Sand I, et al. Detection of subtle gait disturbance and future fall risk in early multiple sclerosis. Neurology. 2020 Mar 31;94(13):e1395-e1406. [PubMed]

2. Laufer $Y$, Hocherman S, Dickstein R. Accuracy of reproducing hand position when using active, compared with passive movement. Physio Therapy Research International. 2001 Jan;16(2): 65-75. [Internet]

3. Aliabadi S, Khanmohammadi R, Olyaei G, Ghotbi N. Comparison of the Position Sense of the Knee Joint in Patients With Multiple Sclerosis and Healthy Controls. JMR. 2019 Nov; 13(1):59-64. [Internet]

4. Stillman B. Making sense of proprioception. The meaning of proprioception, kinaesthesia and related terms. Physiotherapy. 2002;88:66776. [Crossref]

5. Vafadar K, Cote N, Archambault S. Sex differences in the shoulder joint position sense acuity: a cross-sectional study. Musculoskeletal Disord. 2015 Sep 30;16:273-79. [PubMed]

6. Tsvetkova-Gaberska $M$, Pencheva N. The force sense of knee extensors in healthy males and females. Series on Biomechanics. 2019; 33 (3): e3-e13. [Internet]

7. Drouin M, Valovich-mcLeod C, Shultz S, Bruce M, Gansneder J, Perrin D. Reliability and validity of the Biodex system 3 pro isokinetic dynamometer velocity, torque and position measurements. Eur J Appl Physiol. 2004 Jan;91(1):22-9. [PubMed]

8. Han J, Waddington G, Adams R, Anson J, Liu Y. Assessing proprioception: A critical review of methods. $J$ Sport Health Sci. 2016 Mar;5(1):8090. [PubMed]

9. Tsvetkova-Gaberska $\mathbf{M}$ Pencheva N. Assessment of knee joint position sense in healthy males and females. Multidisciplinary $J$ Sci, Edu and Art. 2019: 441-49

10. Wens I, Dalgas U, Vandenabeele F, Krekels M, Grevendonk L, Eijnde B. Multiple sclerosis affects skeletal muscle characteristics. PLoS One. 2014; 9:e108-e158. [PubMed]

11. Dionyssiotis Y. Body composition in multiple sclerosis. Hippokratia. 2013 Jan;17(1):7-11. [PubMed]

12. Chitnis T. The role of testosterone in MS risk and course. Mult Scler. 2018 Jan;24(1):36-41. [PubMed]

13. Niespodzinski B, Kochanowicz A, Mieszkowski J, Piskorska E, Zychowska M. Relationship between joint position sense, force sense, and muscle strength and the impact of gymnastic training on proprioception. Biomed Res Int. 2018 Feb 18; 2018:110. [PubMed]

14. Li L, Ji ZQ, Li YX, Liu W T Correlation study of knee joint proprioception test results using common test methods. J Phys Ther Sci. 2016 Jan; 28(2):478-82. [PubMed]

15. Neamtua M, Neamtub O, Rusub M, Marinc M, Rusub L. Functional muscle balance assessment in multiple sclerosis. J Phys Ther Sci. 2016 Jan; 28(2):478-82. [PubMed]

16. Guney H, Yuksel I, Kaya D, Doral M. The relationship between quadriceps strength and joint position sense, functional outcome and painful activities in patellofemoral pain syndrome. Knee Surg Sports Traumatol Arthrosc. 2016; 24: 2966-2972. [PubMed]

17. Moghadasi A, Ghasemi G, Sadeghi-Demneh E, Etemadifar M. The Effect of Total Body Resistance Exercise on Mobility, Proprioception and Muscle Strength of the Knee in People with Multiple Sclerosis. J Sport Rehabil. 2020 Feb 1;29(2):192-199. [PubMed]

18. Iandolo $\mathrm{R}$, Bommarito $\mathrm{G}$, Falcitano L, Schiavi S, Piaggio N, Mancardi GL, et al. Position Sense Deficits at the Lower Limbs in Early Multiple Sclerosis: Clinical and Neural Correlates. Neurorehabil Neural Repair. 2020 Mar;34(3):260-270. [PubMed].

19. Kalincik T, Vivek V, Jokubaitis $\mathrm{V}$. Sex as a determinant of relapse incidence and progressive course of multiple sclerosis. Brain. 2013 Dec;136(Pt 12):3609-17. [PubMed]

20. Neguesi J, Galamb K, Szilágyi B, Nagatomi R, Hortobágyi T, Tihanyi J. Age-specific modifications in healthy adults' knee joint position sense. Somatosens Mot Res. 2019 Dec;36(4): 262-269. [PubMed] 
Please cite this article as: Tsvetkova-Gaberska M, Pencheva N. Assessment of knee joint position sense in patients with multiple sclerosis. J of IMAB. 2021 Jul-Sep;27(3):3893-3900. DOI: https://doi.org/10.5272/jimab.2021273.3893

Received: 25/02/2021; Published online: 19/08/2021

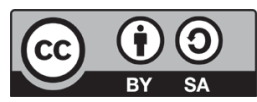

Address for correspondence:

Miglena Tsvetkova-Gaberska

Department of Kinesitherapy, Faculty of Public Health, Health Care and Sports, South-West University "Neofit Rilski”, Blagoevgrad 66, Ivan Mihaylov Str., Blagoevgrad 2700, Bulgaria. E-mail: miglena_tsvetkova@abv.bg 